\title{
Processing of polycyclic aromatic hydrocarbons in evolved planetary nebulae
}

\author{
Ryou Ohsawa $^{1}$, Takashi Onaka ${ }^{1}$, Itsuki Sakon ${ }^{1}$, Issei Yamamura ${ }^{2}$, \\ Mikako Matsuura ${ }^{3}$ and Hidehiro Kaneda ${ }^{4}$ \\ ${ }^{1}$ Department of Astronomy, School of Science, University of Tokyo, \\ 7-3-1 Hongo, Bunkyo-ku, Tokyo 113-0033, Japan \\ email: ohsawa@astron.s.u-tokyo.ac.jp \\ ${ }^{2}$ Japan Aerospace Exploration Agency, Institute of Space and Astronautical Science, \\ 3-1-1 Yoshinodai, Chuo-ku, Sagamihara, Kanagawa 252-5210, Japan \\ ${ }^{3}$ Dept. of Physics \& Astronomy, University College London, \\ Gower Street, London WC1E 6BT, United Kingdom \\ ${ }^{4}$ Graduate School of Science, Nagoya University, \\ Furo-cho, Chikusa-ku, Nagoya, Aichi 464-8602, Japan
}

\begin{abstract}
We investigate the infrared emission bands from Polycyclic Aromatic Hydrocarbons (PAHs) in Galactic planetary nebulae (PNe). PAHs in PNe are assumed to be in transition from circumstellar to interstellar PAHs. We select 15 evolved PNe taking account of effective stellar temperatures and obtain infrared spectra of PNe from AKARI $(2.5-5 \mu \mathrm{m})$ and Spitzer $(5-14 \mu \mathrm{m})$ observations. Their evolutionary phase is estimated using $[\mathrm{SIV}]_{10.51} /[\mathrm{NeII}]_{12.81}$. We find that the near-infrared PAH bands are significantly enhanced along with stellar evolution sequence. We also find that the ratio of 3.4 to $3.3 \mu \mathrm{m}$ bands is enhanced. The enhancement might indicate some chemical processing, such as hydrogenation, on small PAHs.
\end{abstract}

Keywords. planetary nebulae: general, infrared: ISM, molecular processes

Polycyclic Aromatic Hydrocarbons (PAHs) are unique and interesting dust species because they show distinct features in the infrared (hereafter, PAH features), which are sensitive to their local environment and to their chemistry (e.g., Peeters et al. 2002; van Diedenhoven et al. 2004), and we expect a significant difference between the PAH features between in circumstellar and in interstellar environments due to the difference in UV radiation field and density. We present our study of $\mathrm{PAH}$ features in planetary nebula $(\mathrm{PN})$, comparing with local environments within the circumstellar envelope. In this report, we focus on the PAH features around $3 \mu \mathrm{m}$, and investigate the variation in the relative strengths of the $\mathrm{PAH}$ features with nebular environments.

We obtain 2.5-5.0 $\mu \mathrm{m}$ near-infrared spectra of Galactic PNe with the AKARI/IRC. We retrieved 5.5-14.0 $\mu \mathrm{m}$ mid-infrared spectra from the Spitzer Archive. To investigate evolved PNe, we selected PNe whose effective stellar temperatures are higher than 70,000 K. We measured the strengths of emission features by model fit to the spectrum, including the PAH features at 3.3, 3.4, 6.2, 7.7, 8.6, and $11.2 \mu \mathrm{m}$. We estimated the nebular environments using $[\mathrm{SIV}]_{10.51} /[\mathrm{NeII}]_{12.81}$. The relative strengths of the PAH features at 3.3 and $6.2 \mu \mathrm{m}$ to $11.2 \mu \mathrm{m}$ are plotted against $[\mathrm{SIV}]_{10.51} /[\mathrm{NeII}]_{12.81}$ on the upper left and the lower left panels in Fig. 1, respectively. We can find a anti-correlation in the ratio of 3.3 to $11.2 \mu \mathrm{m}$ features. However, there is no apparent correlation in the ratio of 6.6 to $11.2 \mu \mathrm{m}$ features. This is also the case among other mid-infrared PAH features at $6.2-11.2 \mu \mathrm{m}$. The right panel in Fig. 1 displays the intensity ratios of the 3.4 to $3.3 \mu \mathrm{m}$ $\mathrm{PAH}$ features against the line ratios. We can find a clear anti-correlation. 

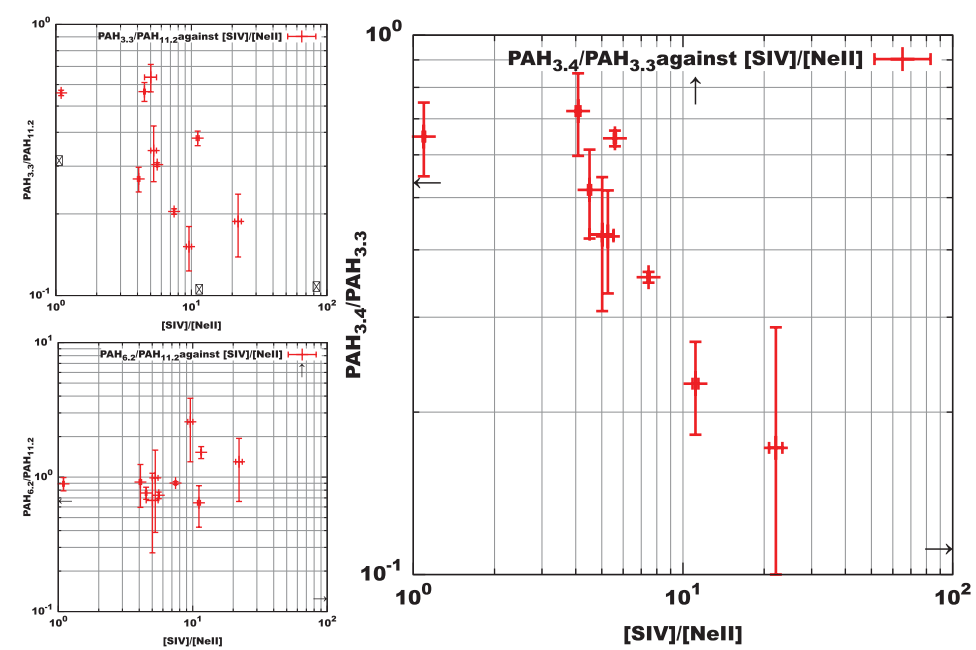

Figure 1. Correlations between the PAH intensity ratios and $[\mathrm{SIV}]_{10.51} /[\mathrm{NeII}]_{12.81}$. The upper left panel shows the ratios of 3.3 to $11.2 \mu \mathrm{m}$ features, while the lower left panel shows those of 6.2 to $11.2 \mu \mathrm{m}$ features. The right panel shows the ratio of 3.4 to $3.3 \mu \mathrm{m}$ features.

Towards the end of PNe evolution, the luminosity of the central star drastically decreases, causing a decrease of $[\mathrm{SIV}] /[\mathrm{NeII}]$. Thus, the present results indicate the selective increase in the near-infrared PAH features and the ratio of the 3.4 to $3.3 \mu \mathrm{m}$ features along with evolution. The decrease in the stellar luminosity also decreases the ionization degrees of PAHs. Change in the ionization degree of PAHs will produce large differences in the PAH features (Allamandola et al. 1989). However, it seems difficult to attribute the variation in the PAH features to the change in the ionization parameter since there is little variations among the mid-infrared PAH features in our data sets. The near-infrared $\mathrm{PAH}$ features are believed to arise from $\mathrm{C}-\mathrm{H}$ bonds in small PAHs. The results might indicate process on $\mathrm{C}-\mathrm{H}$ bonds in small $\mathrm{PAH}$ grains, such as hydrogenation. If the number of hydrogens attached to small PAHs increases, the intensity of the near-infrared PAH features will be enhanced. Although theoretical studies have shown that the number of $\mathrm{H}$-atoms in large PAHs will not vary with environments (Allain et al. 1996; Allamandola et al. 1989), we can still attribute the variation in the near-infrared PAH features to hydrogenation and dehydrogenation processing on small PAH grains. Further studies will reveal what accounts for the variation in the near-infrared $\mathrm{PAH}$ features.

This work is based on observation of AKARI, the JAXA project with the participation of the ESA. We thank all members of the AKARI project for their continuous effort and support. This work uses the Spitzer Space Telescope Archive. We would also express our gratitude to the JPL, Caltech under a contract with NASA. This research was also supported in part by a grant form the Hayakawa Satio Fund awarded by the Astronomical Society of Japan.

\section{References}

Allain, T., Leach, S., \& Sedlmayr, E. 1996, A\&SA 305, 602

Allamandola, L. J., Tielens, A. G. G. M., \& Barker, J. R. 1989, ApJS 71, 733

Peeters, E. S., et al. 2002, A\&SA, 390, 1089

van Diedenhoven, B. E., et al. 2004, ApJ 611, 928 